Int. J. Dev. Biol. 57: 407-414 (2013)

doi: $10.1387 /$ ijdb.130010st

\title{
Hippo signaling components, Mst1 and Mst2, act as a switch between self-renewal and differentiation in Xenopus hematopoietic and endothelial progenitors
}

\author{
SUSUMU NEJIGANE' ${ }^{2}$ SHUJI TAKAHASHI*,2, YOSHIKAZU HARAMOTO, \\ TATSUO MICHIUE ${ }^{1}$ and MAKOTO ASASHIMA ${ }^{3}$
}

\begin{abstract}
${ }^{1}$ Department of Life Sciences (Biology), Graduate School of Arts and Sciences, The University of Tokyo, Japan, ${ }^{2}$ Komaba Organization for Educational Excellence, College of Arts and Sciences, The University of Tokyo, Japan and ${ }^{3}$ Research Center for Stem Cell Engineering (SCRC), National Institute of Advanced Industrial Science and Technology (AIST), Tsukuba, Ibaraki, Japan
\end{abstract}

\begin{abstract}
Hippo signaling is a conserved pathway that regulates cell proliferation and organ size control. Mst1 and Mst2 were identified as homologs of hippo and as core kinases of the Hippo pathway in mammals. Here, we have characterized the role of Mst1 and Mst2 during Xenopus primitive hematopoiesis. We showed that Mst1 and Mst2 were strongly expressed in the Xenopus ventral blood island, where primitive hematopoiesis is initiated. Loss-of-function analysis of Mst1/2 revealed morphogenetic defects, including short axis, smaller eyes and abnormal epidermis, and decreased phosphorylation of Yap. Mst1/2 morphants did not exhibit any change in the expression of hematopoietic and endothelial progenitor markers in early hematopoietic development. In addition, we have shown that such progenitor markers were continuously expressed through to the late hematopoietic development stage. As a result, the expression of erythroid, myeloid and endothelial differentiation markers were decreased in Mst1/2 morphants. Our results indicate that Mst1/2 act as a differentiation switch in Xenopus hematopoietc and endothelial progenitors.
\end{abstract}

KEY WORDS: Hippo signaling, Mst, Yap, primitive hematopoiesis, Xenopus tropicalis

\section{Introduction}

The Hippo signaling pathway plays a key role in regulating cell growth and organ size, and the core components of Hippo signaling are highly conserved from vertebrates to fly (Zhao et al., 2011). In vertebrates, mammalian sterile 20-like kinase 1 and 2 (Mst1/2; homolog of Hippo in fly) are serine/threonine kinases possessing the SARAH (Sav/Rassf/Hippo) domain. Mst1/2 form hetero- and homo-dimers, interacting with Sav1 and Rassf family members, which activate Mst1/2 (Scheel and Hofmann, 2003; Callus et al., 2006; Guo et al., 2007). Mst1/2 are also activated by Tao1 (Poon et al., 2011), a putative upstream regulator, as well as by caspasedependent proteolytic cleavage (Lee et al., 2001), and/or autophosphorylation (Glantschnig et al., 2002). Activated Mst1/2 directly phosphorylate and activate large tumor suppresser homolog 1 and 2 (Lats1/2; homolog of Warts in fly), which are other core kinases in the Hippo signaling pathway (Dong et al., 2007). Phosphorylated Lats1/2 phosphorylate and inhibit Yes-associated protein (Yap; homolog of Yki in fly) and the transcriptional coactivator with PDZ domain (TAZ; ortholog of Yap). The phosphorylation of Yap/TAZ corresponding to serine 127 in human Yap induces binding to 143-3 and consequently, leads to cytoplasmic retention of Yap/TAZ (Zhao et al., 2007; Hao et al., 2008; Lei et al., 2008). In addition, phosphorylation of Yap/TAZ corresponding to serine 381 in human Yap triggers ubiquitination and subsequent degradation (Zhao et al., 2010; Liu et al., 2010). Intact Yap/TAZ proteins are primarily localized in the nucleus, where they interact with transcriptional factors (TFs), including TEAD1-4 (via the TEAD-binding domain of Yap/TAZ), Runx1/2 (via the WW domain), and regulate gene expressions related to growth and proliferation (Zhao et al., 2008;

Abbreviations used in this paper: VBI, ventral blood island.

\footnotetext{
*Address correspondence to: Shuji Takahashi. Komaba Organization for Educational Excellence, College of Arts and Sciences, University ofTokyo, 3-8-1 Komaba, Meguroku, Tokyo 153-8902, Japan. Fax: +81-3-5465-8906.Tel: +81-3-5465-8908. E-mail: shujit@csls.c.u-tokyo.ac.jp

Supplementary Material (two figures) for this paper is available at: http://dx.doi.org/10.1387/ijdb.130010st
}

Accepted: 7 December 2012. Final, author-corrected PDF published online: 15 April 2013. Edited by: Makoto Asashima

ISSN: Online 1696-3547, Print 0214-6282 
Zhang et al., 2009; Yagi et al., 1999; Cui et al., 2003).

In mice, gene ablation of $M s t 1 / 2$ results in death at approximately embryonic day 8.5 with the following phenotypes: severe growth retardation, failed placental development, impaired yolk sac/embryo vascular patterning and primitive hematopoiesis, increased apoptosis in placenta and embryo, and disorganized proliferating cells (Oh et al., 2009). In addition, Yap-depletion in mouse is embryonically lethal due to defective embryonic axis elongation and yolksac vasculogenesis (Morin-Kensicki et al., 2006). These results implicated Hippo signaling in primitive hematopoiesis.

The ontogeny of embryonic hematopoiesis and vasculogenesis, known as primitive hematopoiesis, is conserved in vertebrates (Galloway and Zon, 2003; Medvinsky et al., 2011), in which it usually take place in the blood island. The mammalian and avian blood island is formed in the early yolk sac. In Xenopus embryo, primitive hematopoiesis initiates in the ventral blood island (VBI), which constitutes the anterior $\mathrm{VBI}(\mathrm{aVBI})$ and the posterior VBI $(\mathrm{pVBI})$ domains. Previous attempts to characterize fate-mapping blastomeres of the 32-cell embryo indicated that aVBI derives from the dorsal vegetal blastomeres and that $\mathrm{pVBI}$ derives from the ventral vegetal blastomere (Ciau-Uitz et al., 2000; Lane and Sheets, 2002). The primary site for primitive myeloid cell differentiation is the aVBI, which initially expresses hematopoietic and endothelial progenitor markers, including Scl, Runx1, Lmo2, and Fli1. Subsequently, pVBI expresses several hematopoietic and endothelial
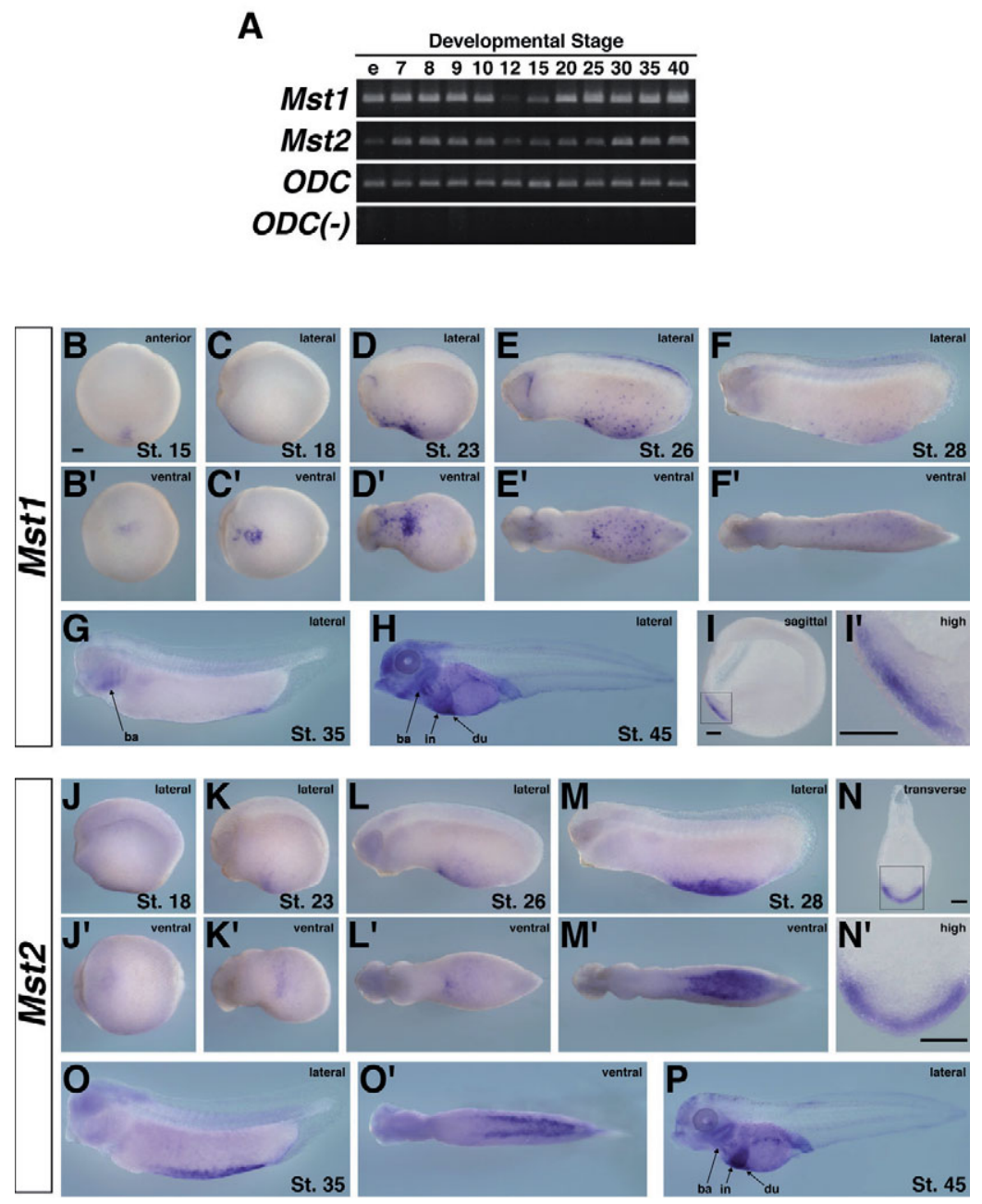

markers and gives rise to embryonic erythrocyte, myeloblast, and vascular endothelial cells (Ciau-Uitz et al., 2010; Liu et al., 2008). It is also known that these hematopoietic lineage and endothelial cells are derived from the common multipotent precursor cells of the hemangioblast (Sabin 1920; Murray, 1932).

Recently, Yap/TAZ was shown to regulate proliferation, selfrenewal, and pluripotency in the following undifferentiated cells: embryonic stem cell (ESC) and induced pluripotent stem cell (iPSC) populations (Lian et al., 2010), intestinal stem cell (ISC) progenitors (Camargo et al., 2007), epidermal progenitors (Zhang et al., 2011), liver progenitors (Camargo et al., 2007; Dong et al., 2007), neural progenitors (Cao et al., 2008), and other stem cells (Hiemer and Varelas, 2012). Hippo signaling plays an important role in the stemness of undifferentiated/progenitor cells as well as organ size control. However, little is known about the role of Hippo signaling in hemangioblast and hematopoietic and endothelial progenitor.

Here, we report that $M s t 1 / 2$ are expressed in VBI, where they act as a switch between self-renewal and differentiation in Xenopus hematopoietic and endothelial progenitor.

\section{Results}

\section{Mst1 and Mst2 expression is localized in the ventral blood island in Xenopus tropicalis embryos}

To determine the spatiotemporal expressions of Mst1/2, we performed semi-quantitative RT-PCR and WISH analyses as the developmental stages progressed. Mst1/2 were continuously expressed throughout the early developmental stages, although Mst1 expression was temporally decreased at the early gastrula stage (Fig. 1A). Wholemount in situ hybridization (WISH) analyses confirmed that these genes were ubiquitously expressed before the neurula stage (data not shown). The localized expression of Mst1 was initially detected in the aVBI at stage 15 (Fig. 1B, B'). Subsequently, Mst1 was expressed in the aVBI and in scattered cells corresponding to migrating myeloblasts, with weak expression also detected in the pVBI (Fig. 1CF,C'-F'). At the tadpole stages, Mst1 was detected in the branchial arches, intestine, and duodenum (Fig. $1 \mathrm{G}, \mathrm{H}$ ). Hemi-sectioning of a stage-18 embryo revealed detailed

Fig. 1. Expression pattern of Mst 1 and Mst2 in Xenopus tropicalis development. (A) RT-PCR analysis revealed the temporal expression patterns of Mst1 and Mst2. RNA was extracted from Xenopus tropicalis embryos at the stages indicated above each lane. ODC were used as internal controls. ODC(-), RT-PCR without reverse transcriptase in ODC reaction. e, unfertilized egg. (B-P) Whole-mount in situ hybridization analysis of Mst1 and Mst2. (B-H) Localized expression pattern of Mst1. (B') The anterior view corresponding to (B). ( $\left.\mathbf{C}^{\prime}-\mathbf{F}^{\prime}\right)$ The ventral view corresponding to C-F. (I) Hemi-sectioning of a stage-18 embryo revealed detailed localization of Mst1 in the aVBI. (I') High magnification view of the boxed region in (I). (J-M,O,P) Localized expression pattern of Mst2. ( $\left.\mathbf{J}^{\prime}-\mathbf{M}^{\prime}, \mathbf{O}^{\prime}\right)$ The ventral view corresponding $(\mathrm{J}-\mathbf{M}, \mathbf{P}, \mathrm{O})$. (N) Transverse sectioning of a stage-28 embryo revealed detailed localization of Mst2 in the pVBI. (N') High magnification view of the boxed region in (N). Scale bars, $100 \mu \mathrm{m}$. View direction is indicated in the upper right of each panel. Developmental stage is indicated in the lower right. Abbreviations: ba, branchial arch; du, duodenum; in, intestine. 
A

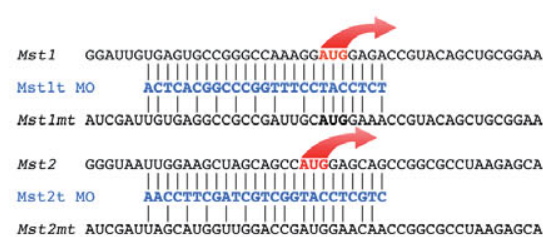

B

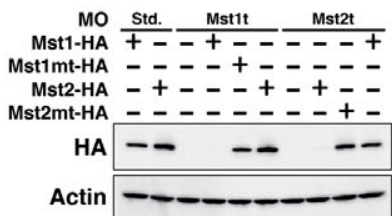

D
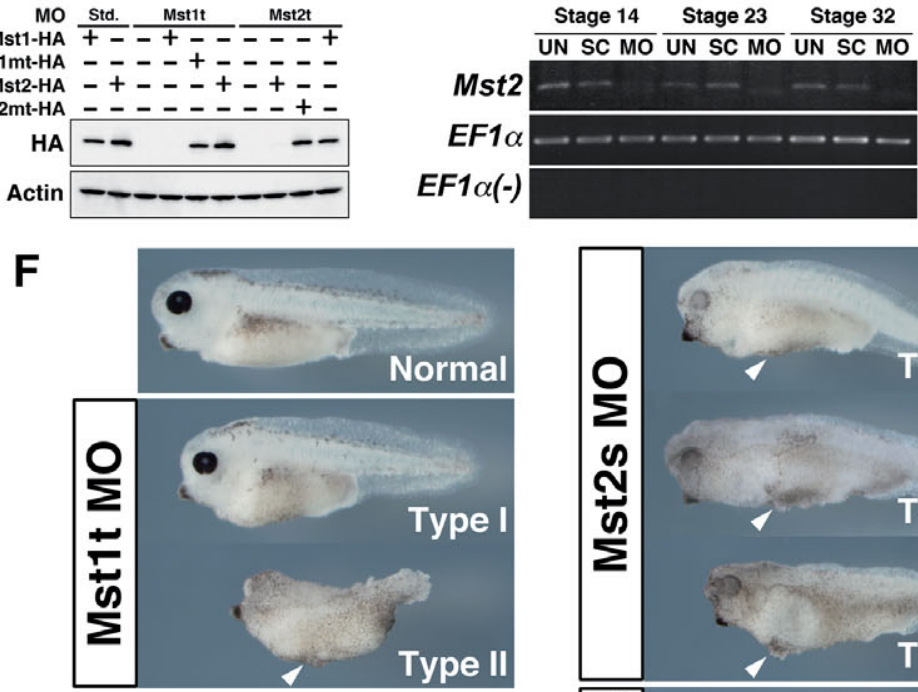

G

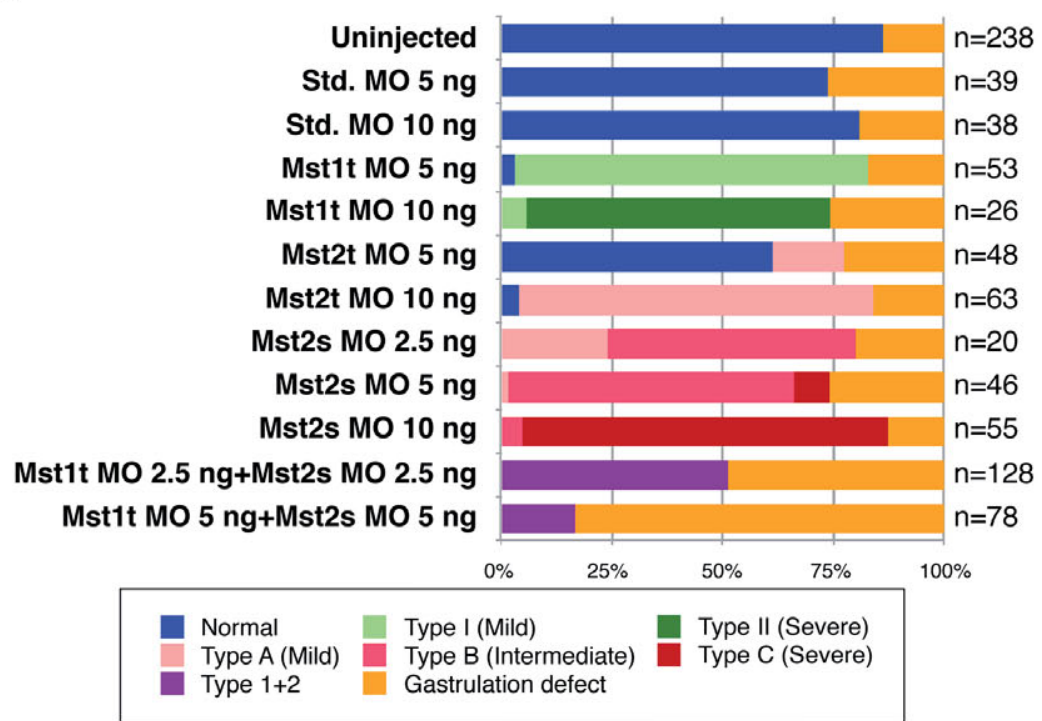

C
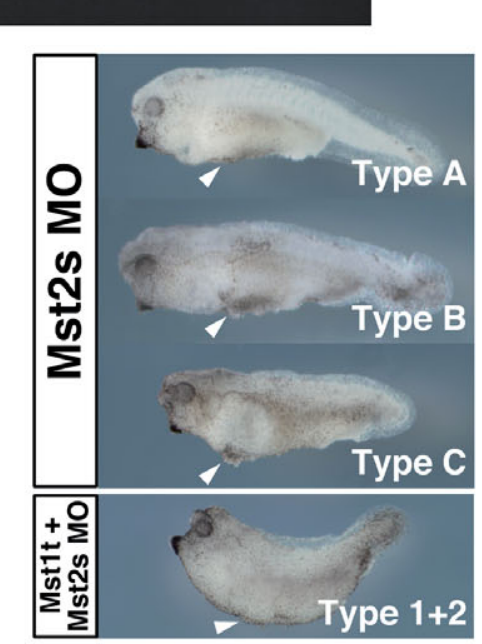$$
\text { . }
$$

E
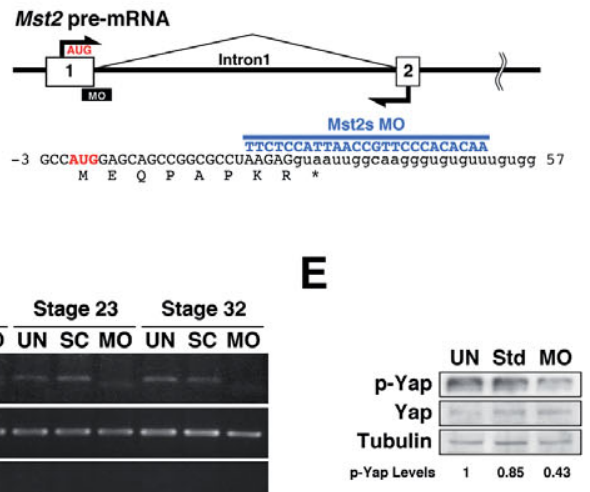

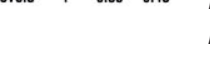


localization of Mst1 in the aVBI (Fig. 1 I,I'). The Mst2 transcripts were weakly expressed in aVBI with a pattern identical to that of Mst1 (Fig. $1 \mathrm{~J}, \mathrm{~J}$ '). Mst2 was weakly expressed in pVBI until the early tailbud stage, after which it was strongly expressed in pVBI from stage 28 until stage 35. (Fig. 1 K-M,K'-M',O,O'). Hemisectioning of a stage-28 embryo revealed detailed localization of Mst2 in the pVBI (Fig. $1 \mathrm{~N}, \mathrm{~N}^{\prime}$ ), and at stage 45, it was detected in the branchial arches, intestine, and duodenum as for Mst1 (Fig. 1P). Thus, Mst1/2 were transiently and specifically expressed in $\mathrm{VBI}$, suggesting that Mst1/2 may function in hematopoiesis and vascular formation.

\section{Mst1 and Mst2 depletion synergistically induced morpho- genic defects}

We generated two kinds of Mst1/2 MOs, the translation-blocking $\mathrm{MO}$, Mst1t $\mathrm{MO}$ and Mst2t MO, and splice-blocking MO, Mst1s $\mathrm{MO}$ and Mst2s MO (Fig. $2 \mathrm{~A}, \mathrm{C}$ ). We first tested whether the translation-blocking MOs could inhibit translation by coinjecting HA-tagged Mst1/2 mRNA including sequences of the AUG initiation codon and 5'UTR to which MO bind (Mst1-HA and Mst2-HA) or mutated sequences of them (Mst1mt-HA and Mst2mt-HA) together with Mst1t MO and Mst2t MO into 2-cell-stage Xenopus laevis embryos. Western blotting analysis performed at stage 10.5 showed that Mst1t MO and Mst2t MO specifically and completely inhibited the translation of Mst1-HA and Mst2-HA respectively, but not of Mst1mt-HA and Mst2mt-HA (Fig. 2B). To address the splice-blocking effects of Mst1s MO and Mst2s MO, we performed RT-PCR analysis of MO-injected Xenopus tropicalis embryos. Mst2s MO (5 ng) efficiently blocked the proper splicing (Fig. 2D), whereas Mst1s MO (10 ng) had no effect on splice blocking (data not shown).

It has reported that phosphorylation level of Yap was significantly reduced by depletion of Mst1 and Mst2 in the mouse liver (Zhou et al., 2009; Song et al., 2010; Lu et al., 2010). We tested whether Yap phosphorylation was decreased in the Mst1/2 double-knockdown embryos with western blots. Compared to standard control MO-injected embryos phospho-Yap level was clearly decreased in the Mst1/2 doubleknockdown embryos (Fig. 2E). These results suggested that Mst1 and Mst2 MO effectively inhibited production of Mst1 and Mst2, resulting in reduction of Yap phosphorylation.

We then performed lossof-function analysis of Mst1/2 using Mst1t MO, Mst2t MO, and Mst2s MO. Mst1-depleted embryos exhibited a short axis and impaired normal morphogenesis including malformation of the ventral epidermis at higher $\mathrm{MO}$ concentrations. (Fig. $2 \mathrm{~F}, \mathrm{G}$ ). Mst2 morphants were severe phenotypes in a concentrationdependent manner, including epidermodysplasia (Fig. 2 F,G), and the embryo phenotypes were equivalent for Mst2t MO-injected and Mst2s MO-injected embryos. In addition, we found that Mst2s MO-injected embryos showed more severe phenotypes than Mst2t MO-injected embryos (Fig. 2G). Based on these results, we used Mst1t MO and Mst2s MO for the subsequent morphant analyses. Mst1/2 double-knockdown embryos showed a shortened body axis phenotype and gastrulation defect in a concentration-dependent manner (Fig. 2 F,G).

\section{Mst1/2 is required for normal formation of embryonic erythrocytes, myeloblasts and vascular endothelium}

As Mst1/2 were specifically expressed in VBI, we next examined the effects of Mst1/2 depletion on embryonic erythrocytes, using globinas a marker of erythrocyte differentiation. Compared to standard control MO (5 ng)-injected embryos, the expression pattern of $\alpha$-globin did not alter in the Mst1 $\mathrm{MO}(5 \mathrm{ng})$-injected embryos (Fig. 3 A,B, A',B'). However, Mst2 and Mst1/2-depleted embryos exhibited decreased $\alpha$-globin expression (Fig. 3 C,D,C', D'). At stage 40 , circulating erythrocytes were observed in the veins of standard control MO-injected embryos, whereas knockdown of Mst1, Mst2 and Mst1/2 inhibited the formation and circulation of erythrocytes (Fig. $3 \mathrm{E}-\mathrm{H}$ ). In addition, o-dianisidine staining to visualize circulating erythrocyte (O'Brien, 1961) showed hardly any staining in the Mst2 and Mst1/2 morphants but not Mst1 morphant, compared to standard control MO-injected embryos (Fig. $3 \mathrm{I}-\mathrm{L}$ ). Moreover, RT-qPCR analysis of $\beta$-globin revealed
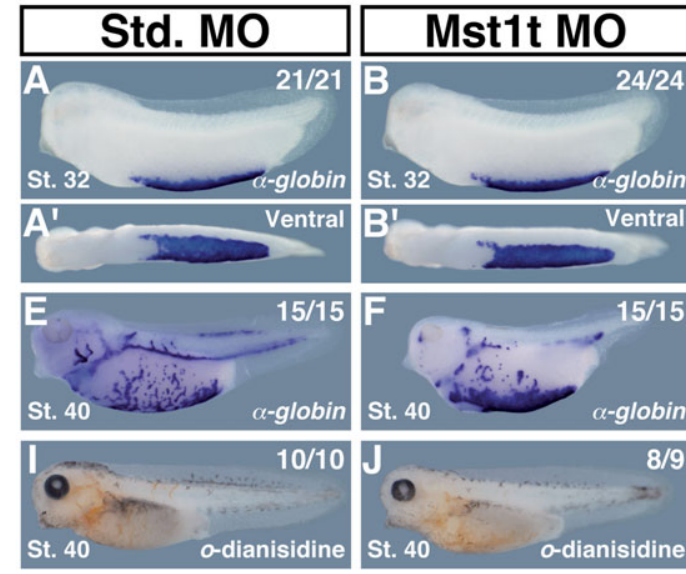

M

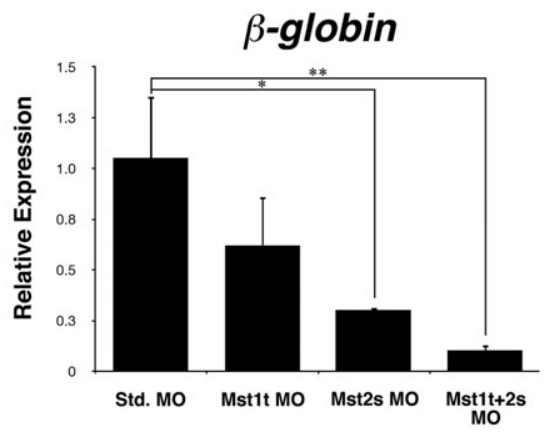

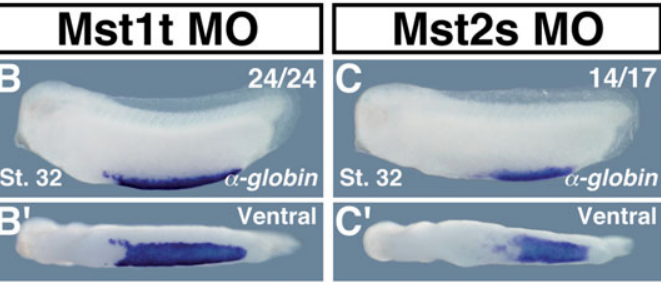
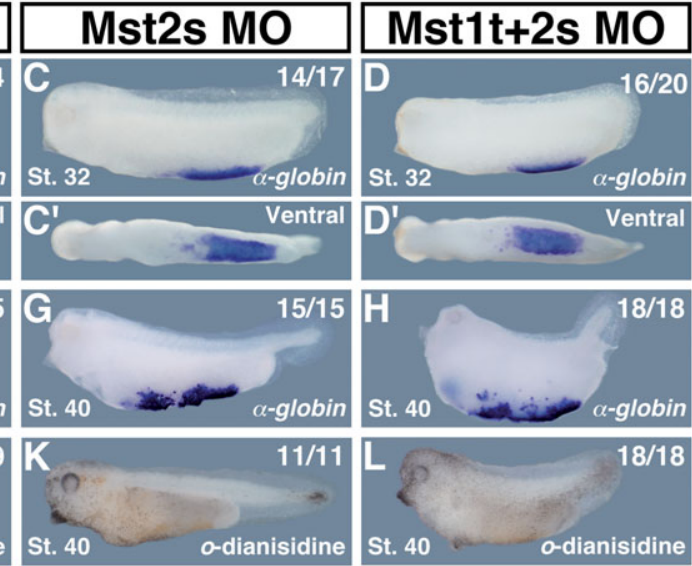

Fig. 3. Effects of Mst1/2 MO injection on embryonic erythrocyte formation. (A, $\left.\mathbf{A}^{\prime}\right)$ Erythrocyte marker, $\alpha$-globin, was normally expressed in the $\mathrm{VBI}$ of embryos injected with standard control MO (Std. MO, 5 ng). (B-D') Knockdown of Mst1 (Mst1t MO, 5 ng), Mst2 (Mst2s MO, $5 \mathrm{ng}$ ) and Mst1/2 (Mst1t+2s MO, 2.5/2.5 ng) decreased $\alpha$-globin expression in VBI. (E) At the later stage, $\alpha$-globin was detected in circulating erythrocytes in the vein of standard control MO-injected embryos. (F-H) Knockdown of Mst1, Mst2 and Mst1/2 inhibited the circulation of erythrocytes. (I) O-dianisidine staining also visualized circulating erythrocytes in controls, (K-L) whereas erythrocytes were hardly visualized in Mst1, Mst2 and Mst1/2 morphants. (M) RT-qPCR analysis revealed that knockdown of Mst $1 / 2$ decreased $\beta$-globin expression. Expression levels were normalized relative to ODC. Values represent means $+S D$ of three independent experiments, ${ }^{*} \mathrm{P}<0.05,{ }^{*} \mathrm{P}<0.01$. 
A

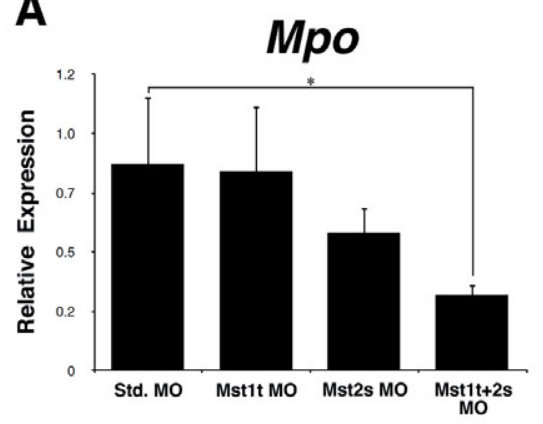

$\mathbf{F}$

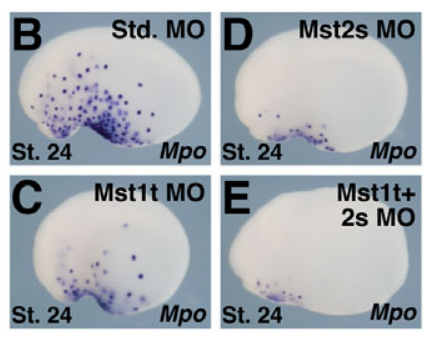

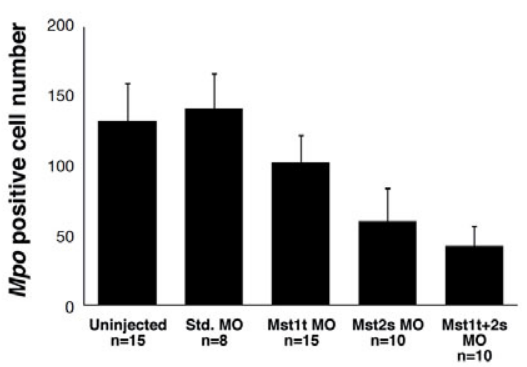

Fig. 4. Effects of Mst1/2 MO injection on embryonic myeloblast formation. (A) Mpo is detected in myeloblast in standard control MO-injected embryo. (B-D) Knockdown of Mst1, Mst2 and Mst1/2 decreased myeloblast numbers, and (E) Mpo-positive cell number was synergistically decreased by Mst1/2 MO injection. (F) RT-qPCR analysis revealed that the knockdown of Mst1/2 decreased expression of Mpo, a myeloblast marker. Values represent means $+S D$ of three independent experiments, ${ }^{*} \mathrm{P}<0.05,{ }^{*} \mathrm{P}<0.01$.

synergistically decreased expression of $\beta$-globin in the Mst morphants (Fig. 3M). We also assessed primitive myelopoiesis following Mst depletion. Expression of myeloblast marker, Mpo, was decreased by Mst depletion (Fig. 4A). WISH analysis of $M p o$ was also revealed that Mpo-positive cell numbers were decreased in Mst1, Mst2 and Mst1/2 MO-injected embryos (Fig. 4 B-F). Furthermore, we found that depletion of Mst impaired appropriate formation of the embryonic vasculature based on a decrease in vascular endothelium markers, Ami, Tie2, and Msr, and abnormal vascular patterning in the Mst morphants (Fig. 5).

To address the specificity of the Mst1/2 knockdown, we assessed whether Mst1 or Mst2 construct could rescue the phenotype and knockdown effects of Mst1/2 morphant. The injection of Mst1 or Mst2 expression constructs, which were driven by EF1 $\alpha$ promoter, rescued morphogenetic abnormality of Mst1/2 knockdown embryo (Supplemental Fig. 2A-E). Furthermore, markedly decreased expression of $\beta$-globin in the Mst $1 / 2$ knockdown embryo was partially rescued by injection of Mst1 or Mst2 DNA (Supplemental Fig. 2F). The disruption of embryonic vascular patterning in the Mst1/2 morphant was also rescued with injection of Mst1 or Mst2 DNA but not LacZ DNA (Supplemental Fig. 2 G-J). These results suggested that Mst1/2 are required for erythropoiesis, myelopoiesis, and vascular formation.
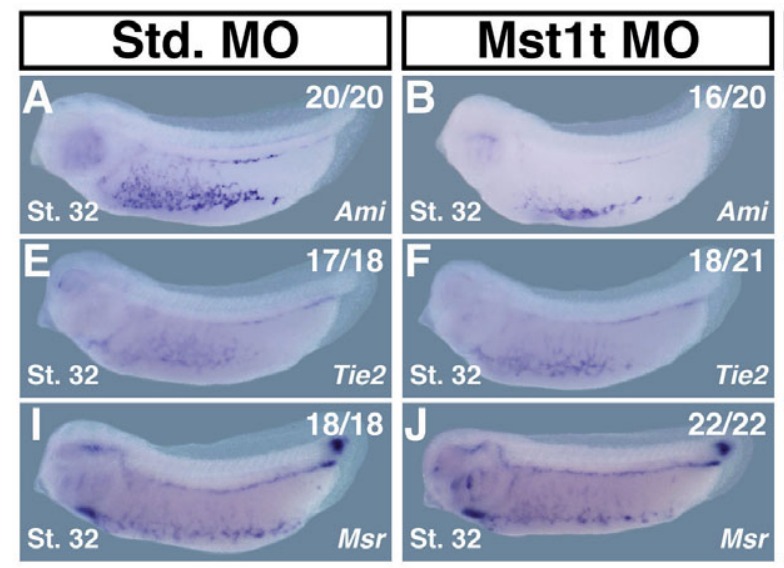

Mst1/2 depletion promotes maintenance of the hematopoietic and endothelial progenitor

The hemangioblast is a common precursor of hematopoietic and endothelial lineages (Orkin and Zon, 2008), and the Mst1/2-depleted embryos studied here exhibited defects in both hematopoietic cells and endothelial cells. Therefore, we examined whether hematopoietic and endothelial progenitor, and/or early differentiation markers, Scl, Runx1, Lmo2, Gata2, C/EBP $\alpha$, Spib, Gata1, Fli1, and Etv2 were affected by depletion of Mst1/2. In the early tailbud stage, the early hematopoietic and endothelial progenitor marker expressions were not affected (Fig. $6 \mathrm{~A}-\mathrm{H}$ ). In aVBI of same stage and neurula stage, WISH analyses of $C / E B P \alpha$ and Spib, which were myeloid progenitor markers (Chen et al., 2009), revealed that expression of these markers were maintained, but migrating myeloid cells were obviously decreased in the Mst1/2 knockdown embryo (Fig. 6 I-N, Fig. 4). At the early tadpole stage, these markers were gradually downregulated in standard control MO-injected embryos as cells differentiated into hemocytes and endothelia, whereas Mst1/2knockdown embryos maintained the earlier expression levels of these markers (Fig. $6 \mathrm{O}-\mathrm{V}$ ). In this stage, Gata1 is one of the most important transcriptional factors for erythroid differentiation (Pevny et al., 1991), under the regulation of Gata2 (Bresnick et al., 2010). Depletion of Mst1/2 decreased expression of Gata1 in VBI (Fig. 6

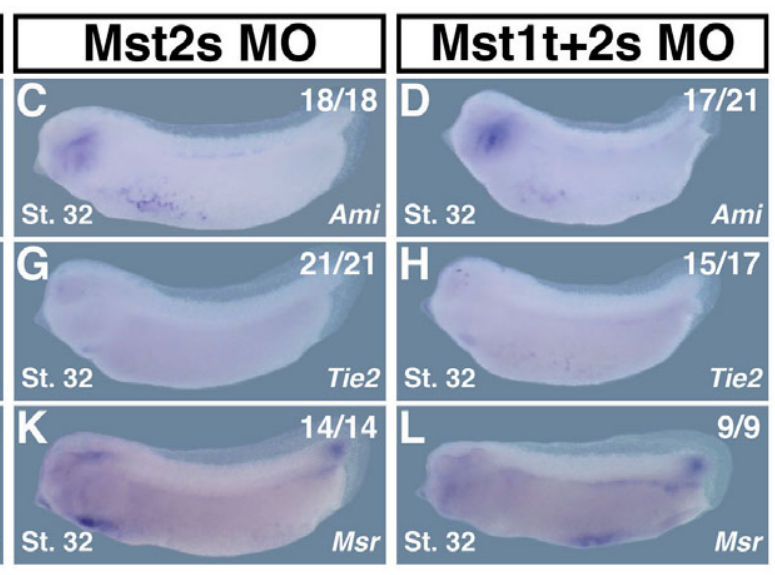

Fig. 5. Effects of Mst1/2 MO injection on embryonic vascular plexus formation. WISH analysis of embryonic vascular markers, Ami, Tie2, and Msr. (A,E,I) The embryonic vascular network had started forming at stage 32 in standard control MO-injected embryo, (B-D,F-H,J-L) and this was disrupted by Mst1, Mst2, and Mst1/2 knockdown. Sample numbers are indicated in the upper right of each panel. Developmental stage is indicated in the lower left. Assessed marker is indicated in the lower right all panels. 

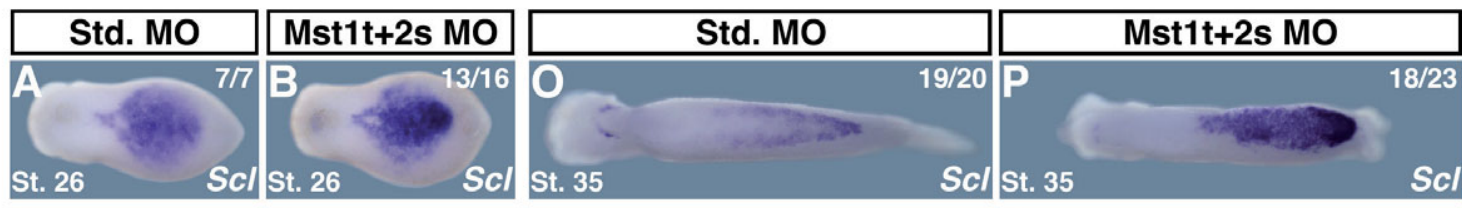

Fig. 6. Effects of Mst1/2 MO injection on hematopoietic and endothelial progenitors. (A-H) At the early stage, no differences in the expression of hematopoietic and endothelial progenitor markers, Scl, Runx1, Lmo2, and Gata2, were observed between standard control MO (Std. MO, 5 ng)- and Mst1/2 MO (Mst1t+2s MO, 2.5/2.5 ng)-injected embryos. (I-N) In the Mst1/2 morphant, expressions of early myeloid progenitor marker, C/EBP $\alpha$ and Spib were maintained in aVBI, but reduction of migrating differentiated myeloid cells was observed. Arrow indicate migrating myeloid cells from aVBI. (O-V) At the later stage, following the differentiation of hematopoietic cells, Scl, Runx1, Lmo2, and Gata2 expression gradually decreased in the VBI of standard controlMO-injected embryos, whereas these markers were continuously expressed at similar levels
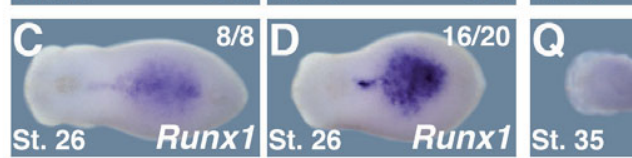

Sc/ St. 35

$\mathrm{Scl}$
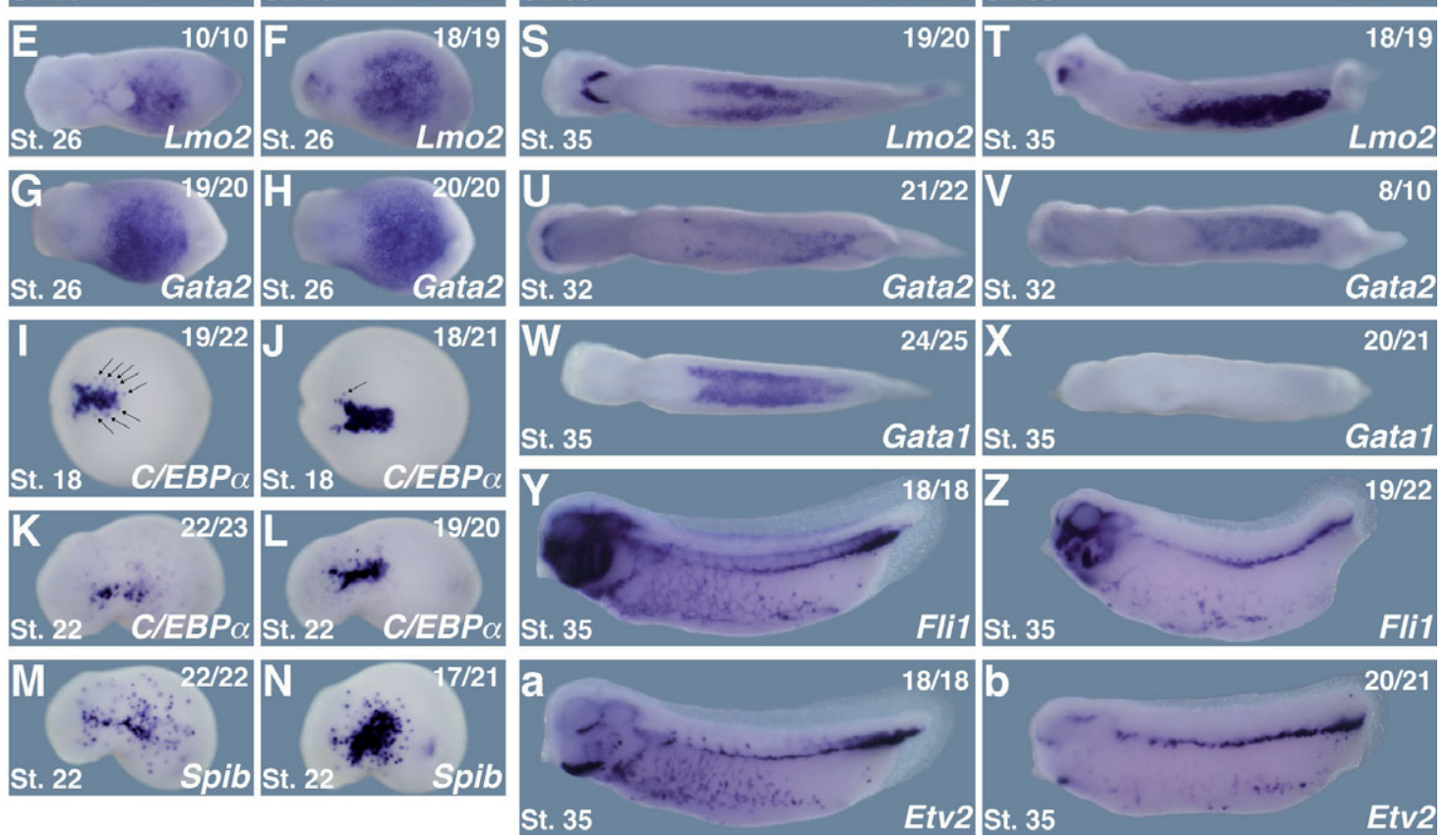

in the Mst1/2 knockdown embryo. (W,X) The pre-erythroblast marker, Gata1, was markedly decreased by Mst1/2 knockdown. (Y-b) The expression patterns of the endothelial progenitor marker, Fli1 and Etv2, were disrupted by depletion of Mst1/2. Sample numbers are indicated in the upper right of each panel, and developmental stage is indicated in the lower left. Assessed marker is indicated in the lower right of each panel.

$\mathrm{W}, \mathrm{X}$ ), as well as the early endothelial markers, Fli1 and Etv2 (Fig. 6 Y-b). Thus, Mst1/2 play an important role in cell differentiation from hematopoietic and endothelial progenitors.

\section{Discussion}

\section{Mst1/2 expression level regulates Hippo signaling}

Our experiments revealed that Mst $1 / 2$ are transiently and locally expressed in VBI (Fig. 1). Loss-of-function experiment showed that decrease of phosphorylation of Yap protein (Fig. 2E). This is the first report that expression levels of Hippo signal transducers regulate the lineage-specific intensity of signal transduction. Previous studies have shown that TFs expressed in hemangioblast and $\mathrm{HSC} /$ early hematopoietic and endothelial lineages, such as Scl, Runx1, Lmo2, and Gata2, form a robust transcriptional regulatory network (Wilson et al., 2010). Additionally, stage-specific transcriptional programs control hematopoietic gene expression and the subsequent differentiation into multiple mature hematopoietic lineages (Pal et al., 2004; Orkin and Zon, 2008; Monteiro et al., 2011). The difference in expression patterns of Mst1 and Mst2 suggest individual regulation via distinct hematopoietic lineagespecific TFs. In fact, the binding sites for various hematopoietic TFs that are highly expressed in pVBI, including Gata, Ets, Runx, and Scl, are conserved in the Mst2 gene promoter region, but not in the equivalent regions of the Mst1 gene, which is not highly expressed in $\mathrm{pVBI}$ (data not shown). Such programmed and localized expression of Mst1/2 resulted in enhanced Hippo signaling in VBI. Although Tao1 is known to directly phosphorylate Mst and promote Hippo pathway activation in mammalian cells (Poon et al., 2011), it remains less clear how the pathway is regulated upstream of Mst.

\section{Hippo signaling acts as a switch between self-renewal and differentiation in hematopoietic and endothelial progenitors}

In this study, we found Mst1/2 to be transiently and locally expressed in VBI in a hematopoietic differentiation stage-progressive manner (Fig. 1), and revealed that Mst1/2 double knockdown strikingly disrupted erythropoiesis, myelopoiesis, and vasculogeneis and maintained stem cell or progenitor population (Fig. 3-6). Due to the disruption of hippo signaling in the Mst1/2 knockdown embryo, activated Yap/TAZ may inhibit hematopoietic and endothelial differentiation. Consistent with this finding, previous studies demonstrated that Yap/TAZ regulates proliferation, self-renewal, and pluripotency in several stem/progenitor cells (Hiemer and Varelas, 2012). In mouse intestine, Yap expression was restricted in undifferentiated/progenitor cells and Yap overexpression induced loss of differentiation and expansion of progenitor cells (Camargo et al., 2007). In addition, Mst1/2 restrained intestinal stem cell proliferation by inhibiting the nuclear localization of Yap (Zhou 
et al., 2011). It seems quite probable that the same mechanism of Yap regulation could act to control stemness in hematopoietic and endothelial progenitors. Our preliminary data showed that besides decreased expression of embryonic erythrocyte and vascular marker and network-dissipated abnormal vasculature, decreased stem cell marker were observed in the Yap morphant (data not shown). These observations suggested that Yap was also required progenitor to proliferate in the early stage. Furthermore, our previous study showed that Yap expression is transiently suppressed in VBI at stage 30, the most prominent blood-producing stage (Nejigane et al., 2011), suggesting that Yap is also regulated at the transcriptional level in VBI. Taken together, the evidence indicates that Yap is required but transient elimination of both Yap protein and transcript in hematopoietic and endothelial progenitors is necessary during hematopoietic and endothelial differentiation.

Our study demonstrated that Hippo signaling components participated in hematopoietic and endothelial progenitors regulation in Xenopus embryogenesis. These finding is important for identifying potential therapeutic targets of hematopoietic and vascular diseases.

\section{Materials and Methods}

\section{Plasmid construction}

Using JGI Xenopus tropicalis v4.1, we searched and identified the genomic clones corresponding to Xenopus tropicalis Mst1 and Mst2. The full-length ORFs of Mst1 and Mst2 were then amplified by PCR from the cDNA of Xenopus tropicalis gastrula-stage embryos. Primer sequences are shown in the supplementary table. Each clone was subcloned into the pCS2p (+) vector, which was digested by Stul. pCS2p-Mst1 and pCS2pMst2 (Genbank Accession No. AB744231 and AB744232, respectively) were used for synthesis of the in situ hybridization probes and capped mRNAs. MacVector software (MacVector Inc) was used for multiple protein sequence alignment and phylogenetic tree analysis of the Mst genes (see Supplemental Fig. 1). HA-tagged Mst1/2 including either the 5'-untranslated region (UTR) to which morpholino antisense oligos (MO) could bind or a mutated 5'UTR to which MO could not bind were amplified by PCR using pCS2p-Mst1 and pCS2p-Mst2, respectively, which were digested by Clal and subcloned into the equivalent sites of the pCS2-HA vector (Nitta et al., 2004). The rescue analyses were performed using pENL, pE-Mst1, and $\mathrm{pE}-$ Mst2, which were LacZ, Mst1, and Mst2 driven by Chick EF1 $\alpha$ promoter, respectively.

\section{Xenopus embryo manipulation, mRNA synthesis for microinjection, and morpholino antisense oligos}

Xenopus embryos were obtained by artificial fertilization according to the method of Ariizumi et al., (Ariizumi et al., 2009). Embryos were cultured in $10 \%$ Steinberg's solution and staged according to the scheme of Nieuwkoop and Faber (Nieuwkoop and Faber, 1994). Capped mRNAs were synthesized using an mMESSAGE mMACHINE SP6 Kit (Life Technologies) according to the manufacturer's instructions. Mst1 and Mst2 translational blocking MOs (Mst1t MO, 5'-TCTCCATCCTTTGGCCCGGCACTCA-3'; Mst2t MO, 5'-CTGCTCCATGGCTGCTAGCTTCCAA-3'; Gene Tools, LLC) were targeted to the 5'UTR including the start codons of Mst1 and Mst2 transcripts, respectively. The splice site-targeted Mst2 MO (Mst2s MO, 5'-AACACACCCTTGCCAATTACCTCTT-3') was designed against the first exon-intron boundary of Mst2 pre-mRNA (the underlining indicates the exonic region). The standard control MO (Gene Tools, LLC) was used as a control.

\section{Whole-mount in situ hybridization and o-dianisidine staining}

Digoxigenin (DIG)-labeled anti-sense RNA probes were synthesized by T7 or SP6 RNA polymerase (Promega) according to the manufacturer's instructions. WISH analysis was performed as described previously (Harland, 1991). After WISH, embryos were embedded in $3 \%$ low melting point agarose $/ 3 \%$ sucrose and sectioned. To synthesize RNA probes, $\alpha-$ globin, Mpo, Ami, Tie2, Msr, C/EBP $\alpha$, Spib, Scl, Runx1, Lmo2, Gata2, C/EBP $\alpha$, Spib,Gata1, Fli1, and Etv2 were amplified by PCR from the cDNA of the tailbud-stage embryos and subcloned into vectors. Primer sequences and vectors are shown in the supplementary table (see supplementary table). These sequences were verified by $A B I$ PRISM ${ }^{\circledR}$ Genetic Analyzer 310 (Applied Biosystems). O-dianisidine staining was performed as described previously (O’Brien, 1961).

\section{$R T-P C R, R T-q P C R$}

Total RNA samples were isolated using RNAiso Plus (Takara Bio Inc), and $1 \mu \mathrm{g}$ of total RNA was used as the template for first-strand cDNA synthesis using SuperScript II Reverse Transcriptase according to the manufacturer's instructions (Invitrogen). The cDNA (1 $\mu \mathrm{l})$ was used as a template for PCR, and quantitative RT-PCR (RT-qPCR) was performed using a StepOnePlus ${ }^{\mathrm{TM}}$ Real-time PCR system (Applied Biosystems) and the Fast SYBR Green Master Mix (Applied Biosystems). Primer sequences are shown in the supplementary table (see Supplemental table). Ornithine decarboxylase $(O D C)$ and elongation factor $1 \alpha(E F 1 \alpha)$ were used as internal controls.

\section{Western blotting}

Western blotting was performed by a standard protocol. Briefly, embryos were harvested at stage 11 or stage 30 and lysed in RIPA buffer containing a Complete Protease Inhibitor Cocktail (Roche). The equivalent of one embryo was loaded and run on a sodium dodecyl sulfate-polyacrylamide gel electrophoresis gel. After electrophoresis, proteins were transferred to Amersham Hybond-P PDVF Membrane (GE Healthcare), and immunostained by anti-HA (F-7; Santa Cruz), anti-Yap (H-9; Santa Cruz) anti-pYap (4912; Cell Signaling Technology), anti-actin (AC-40; SIGMA) and antiTubulin (T3526; SIGMA) as primary antibodies. Phospho-Yap levels were calculated by gel densitometry using ImageJ software (http://rsbweb.nih. gov/ij/), normalizing against Yap levels.

\section{Acknowledgements}

We thank Dr David L. Turner for the generous gift of pCS2 vector. Xenopus tropicalis was provided by the National Bio-Resource Project (NBRP) of the MEXT, Japan. This work was supported by JSPS KAKENHI Grant Number 2459032.

\section{References}

ARIIZUMI, T., TAKAHASHI, S., CHAN, T.C., ITO, Y., MICHIUE, T. and ASASHIMA, M. (2009). Isolation and differentiation of Xenopus animal cap cells. Curr Protoc Stem Cell Biol Chapter 1: Unit 1D.5.

BRESNICK, E.H., LEE, H.Y., FUJIWARA, T., JOHNSON, K.D. and KELES, S. (2010). GATA switches as developmental drivers. J Biol Chem 285: 31087-31093.

CALLUS, B.A., VERHAGEN, A.M. and VAUX, D.L. (2006). Association of mammalian sterile twenty kinases, Mst1 and Mst2, with hSalvador via C-terminal coiled-coil domains, leads to its stabilization and phosphorylation. FEBS J 273: 4264-4276.

CAMARGO, F.D., GOKHALE, S., JOHNNIDIS, J.B., FU, D., BELL, G.W., JAENISCH R. and BRUMMELKAMP, T.R. (2007). YAP1 increases organ size and expands undifferentiated progenitor cells. Curr Biol 17: 2054-2060.

CAO, X., PFAFF, S.L. and GAGE, F.H. (2008). YAP regulates neural progenitor cell number via the TEA domain transcription factor. Genes Dev 22: 3320-3334

CHEN, Y., COSTA, R.M., LOVE, N.R., SOTO, X., ROTH, M., PAREDES, R. and AMAYA, E. (2009). C/EBPalpha initiates primitive myelopoiesis in pluripotent embryonic cells. Blood 114: 40-48.

CIAU-UITZ, A., LIU, F. and PATIENT, R. (2010). Genetic control of hematopoietic development in Xenopus and zebrafish. Int J Dev Biol 54: 1139-1149.

CIAU-UITZ, A., WALMSLEY, M. and PATIENT, R. (2000). Distinct origins of adult and embryonic blood in Xenopus. Cell 102: 787-796 
CUI, C.B., COOPER, L.F., YANG, X., KARSENTY, G. and AUKHIL, I. (2003). Transcriptional coactivation of bone-specific transcription factor $\mathrm{Cbfa} 1$ by TAZ. Mol Cell Biol 23: 1004-1013.

DONG, J., FELDMANN, G., HUANG, J., WU, S., ZHANG, N., COMERFORD, S.A., GAYYED, M.F., ANDERS, R.A., MAITRA, A. and PAN, D. (2007). Elucidation of a universal size-control mechanism in Drosophilaand mammals. Cell130:1120-1133.

GALLOWAY, J.L. and ZON, L.I. (2003). Ontogeny of hematopoiesis: examining the emergence of hematopoietic cells in the vertebrate embryo. Curr Top Dev Biol 53: $139-158$.

GLANTSCHNIG, H., RODAN, G.A. and RESZKA, A.A. (2002). Mapping of MST1 kinase sites of phosphorylation. Activation and autophosphorylation. J Biol Chem 277: 42987-42996.

GUO, C., TOMMASI, S., LIU, L., YEE, J.K., DAMMANN, R. and PFEIFER, G.P. (2007). RASSF1A is part of a complex similar to the Drosophila Hippo/Salvador/ Lats tumor-suppressor network. Curr Biol 17: 700-705.

HAO, Y., CHUN, A., CHEUNG, K., RASHIDI, B. and YANG, X. (2008). Tumor suppressor LATS1 is a negative regulator of oncogene YAP. J Biol Chem 283: 5496-5509.

HARLAND, R.M. (1991). In situ hybridization: an improved whole-mount method for Xenopus embryos. Methods Cell Biol 36: 685-695.

HIEMER, S.E. and VARELAS, X. (2013). Stem cell regulation by the Hippo pathway. Biochim Biophys Acta 1830: 2323-2334.

JANSSON, L. and LARSSON, J. (2012). Normal hematopoietic stem cell function in mice with enforced expression of the Hippo signaling effector YAP1. PLOS One 7: e32013.

LANE, M.C. and SHEETS, M.D. (2002). Primitive and definitive blood share a common origin in Xenopus: a comparison of lineage techniques used to construct fate maps. Dev Biol 248: 52-67.

LEE, K.K., OHYAMA, T., YAJIMA, N., TSUBUKI, S. and YONEHARA, S. (2001). MST, a physiological caspase substrate, highly sensitizes apoptosis both upstream and downstream of caspase activation. J Biol Chem 276: 19276-19285.

LEI, Q.Y., ZHANG, H., ZHAO, B., ZHA, Z.Y., BAI, F., PEI, X.H., ZHAO, S., XIONG, Y. and GUAN, K.L. (2008). TAZ promotes cell proliferation and epithelial-mesenchymal transition and is inhibited by the hippo pathway. Mol Cell Biol 28: 2426-2436.

LIAN, I., KIM, J., OKAZAWA, H., ZHAO, J., ZHAO, B., YU, J., CHINNAIYAN, A., ISRAEL, M.A., GOLDSTEIN, L.S., ABUJAROUR, R. et al., (2010). The role of YAP transcription coactivator in regulating stem cell self-renewal and differentiation. Genes Dev 24: 1106-1118.

LIU, C.Y., ZHA, Z.Y., ZHOU, X., ZHANG, H., HUANG, W., ZHAO, D., LI, T., CHAN, S.W., LIM, C.J., HONG, W. et al., (2010). The hippo tumor pathway promotes TAZ degradation by phosphorylating a phosphodegron and recruiting the SCF ${ }^{\beta-T C P} E 3$ ligase. J Biol Chem 285: 37159-37169.

LIU, F., WALMSLEY, M., RODAWAY, A. and PATIENT, R. (2008). Fli1 acts at the top of the transcriptional network driving blood and endothelial development. Curr Biol 18: 1234-1240.

LU, L., LI, Y., KIM, S.M., BOSSUYT, W., LIU, P., QIU, Q., WANG, Y., HALDER, G., FINEGOLD, M.J., LEE, J.S. et al., (2010). Hippo signaling is a potent in vivo growth and tumor suppressor pathway in the mammalian liver. Proc Natl Acad Sci USA 107: 1437-1442.

MEDVINSKY, A., RYBTSOV, S. and TAOUDI, S. (2011). Embryonic origin of the adult hematopoietic system: advances and questions. Development 138: 1017-1031.

MONTEIRO, R., POUGET, C. and PATIENT, R. (2011). The gata1/pu. 1 lineage fate paradigm varies between blood populations and is modulated by tif1 $\gamma$. EMBO $J$ 30: 1093-1103.

MORIN-KENSICKI, E.M., BOONE, B.N., HOWELL, M., STONEBRAKER, J.R., TEED, J., ALB, J.G., MAGNUSON, T.R., O'NEAL, W. and MILGRAM, S.L. (2006). Defects in yolk sac vasculogenesis, chorioallantoic fusion, and embryonic axis elongation in mice with targeted disruption of Yap65. Mol Cell Biol 26: 77-87.

MURRAY, P.D.F. (1932). The development in vitro of the blood of the early chick embryo. Proc R Soc Lond 11: 497-521.

NEJIGANE, S., HARAMOTO, Y., OKUNO, M., TAKAHASHI, S. and ASASHIMA, M. (2011). The transcriptional coactivators Yap and TAZ are expressed during early Xenopus development. Int J Dev Biol 55: 121-126.

NIEUWKOOP, P.D. and FABER, J. (1994). Normal Table of Xenopus laevis (Daudin). Garland Publishing Inc, New York.
NITTA, K.R., TANEGASHIMA, K., TAKAHASHI, S. and ASASHIMA, M. (2004). XSIPis essential for early neural gene expression and neural differentiation by suppression of BMP signaling. Dev Biol 275: 258-267.

O'BRIEN, B.R.A. (1961). Identification of Haemoglobin by its Catalase Reaction with Peroxide and o-Dianisidine. Biotechnic \& Histochem. 36: 57-61.

OH, S., LEE, D., KIM, T., KIM, T.S., OH, H.J., HWANG, C.Y., KONG, Y.Y., KWON, K.S and LIM, D.S. (2009). Crucial role for Mst1 and Mst2 kinases in early embryonic development of the mouse. Mol Cell Biol 29: 6309-6320.

ORKIN, S.H. and ZON, L.I. (2008). Hematopoiesis: an evolving paradigm for stem cell biology. Cell 132: 631-644.

PAL, S., CANTOR, A.B., JOHNSON, K.D., MORAN, T.B., BOYER, M.E., ORKIN, S.H. and BRESNICK, E.H. (2004). Coregulator-dependent facilitation of chromatin occupancy by GATA-1. Proc Natl Acad Sci USA 101: 980-985.

PEVNY, L., SIMON, M.C., ROBERTSON, E., KLEIN, W.H., TSAI, S.F., D'AGATI, V., ORKIN, S.H. and COSTANTINI, F. (1991). Erythroid differentiation in chimaeric mice blocked by a targeted mutation in the gene for transcription factor GATA-1. Nature 349: 257-260.

POON, C.L., LIN, J.I., ZHANG, X. and HARVEY, K.F. (2011). The sterile 20-like kinase Tao-1 controls tissue growth by regulating the Salvador-Warts-Hippo pathway. Dev Cell 21: 896-906

SABIN, F.R. (1920). Studies on the origin of blood vessels and of red corpuscles as seen in the living blastoderm of the chick during the second day of incubation contributions to embryology. Contrib Embryol 9: 213-262.

SCHEEL, H. and HOFMANN, K. (2003). A novel interaction motif, SARAH, connects three classes of tumor suppressor. Curr Biol 13: R899-900.

SONG, H., MAK, K.K., TOPOL, L., YUN, K., HU, J., GARRETT, L., CHEN, Y., PARK O., CHANG, J., SIMPSON, R.M. et al., (2010). Mammalian Mst1 and Mst2 kinases play essential roles in organ size control and tumor suppression. Proc Natl Acad Sci USA 107: 1431-1436.

WILSON, N.K., FOSTER, S.D., WANG, X., KNEZEVIC, K., SCHÜTTE, J., KAIMAKIS P., CHILARSKA, P.M., KINSTON, S., OUWEHAND, W.H., DZIERZAK, E. et al., (2010). Combinatorial transcriptional control in blood stem/progenitor cells: genomewide analysis of ten major transcriptional regulators. Cell Stem Cell 7: 532-544.

YAGI, R., CHEN, L.F., SHIGESADA, K., MURAKAMI, Y. and ITO, Y. (1999). A WW domain-containing yes-associated protein (YAP) is a novel transcriptional coactivator. EMBO J 18: 2551-2562.

ZHANG, H., LIU, C.Y., ZHA, Z.Y., ZHAO, B., YAO, J., ZHAO, S., XIONG, Y., LEI, Q.Y and GUAN, K.L. (2009). TEAD transcription factors mediate the function of TAZ in cell growth and epithelial-mesenchymal transition. J Biol Chem284: 13355-13362.

ZHANG, H., PASOLLI, H.A. and FUCHS, E. (2011). Yes-associated protein (YAP) transcriptional coactivator functions in balancing growth and differentiation in skin. Proc Natl Acad Sci USA 108: 2270-2275.

ZHAO, B., LI, L., TUMANENG, K., WANG, C.Y. and GUAN, K.L. (2010). A coordinated phosphorylation by Lats and $C K 1$ regulates YAP stability through $S C F^{\beta-T R C P}$. Genes Dev 24: 72-85.

ZHAO, B., TUMANENG, K. and GUAN, K.L. (2011). The Hippo pathway in organ size control, tissue regeneration and stem cell self-renewal. Nat Cell Biol 13: 877-883.

ZHAO, B., WEI, X., LI, W., UDAN, R.S., YANG, Q., KIM, J., XIE, J., IKENOUE, T. YU, J., LI, L. et al., (2007). Inactivation of YAP oncoprotein by the Hippo pathway is involved in cell contact inhibition and tissue growth control. Genes Dev 21 2747-2761.

ZHAO, B., YE, X., YU, J., LI, L., LI, W., LI, S., YU, J., LIN, J.D., WANG, C.Y., CHINNAIYAN, A.M. et al., (2008). TEAD mediates YAP-dependent gene induction and growth control. Genes Dev 22: 1962-1971.

ZHOU, D., CONRAD, C., XIA, F., PARK, J.S., PAYER, B., YIN, Y., LAUWERS, G.Y. THASLER, W., LEE, J.T., AVRUCH, J. et al., (2009). Mst1 and Mst2 maintain hepatocyte quiescence and suppress hepatocellular carcinoma development through inactivation of the Yap1 oncogene. Cancer Cell 16: 425-438.

ZHOU, D., ZHANG, Y., WU, H., BARRY, E., YIN, Y., LAWRENCE, E., DAWSON, D., WILLIS, J.E., MARKOWITZ, S.D., CAMARGO, F.D. et al., (2011). Mst1 and Mst2 protein kinases restrain intestinal stem cell proliferation and colonic tumorigenesis by inhibition of Yes-associated protein (Yap) overabundance. Proc Natl Acad Sci USA 108: E1312-1320. 


\section{Further Related Reading, published previously in the Int. J. Dev. Biol.}

The transcriptional coactivators Yap and TAZ are expressed during early Xenopus development

Susumu Nejigane, Yoshikazu Haramoto, Makoto Okuno, Shuji Takahashi and Makoto Asashima Int. J. Dev. Biol. (2011) 55: 121-126

\section{Hematopoietic development in the zebrafish}

Elizabeth J. Paik and Leonard I. Zon

Int. J. Dev. Biol. (2010) 54: 1127-1137

Primitive and definitive erythropoiesis in the yolk sac: a bird's eye view Guojun Sheng

Int. J. Dev. Biol. (2010) 54: 1033-1043

Genetic control of hematopoietic development in Xenopus and zebrafish Aldo Ciau-Uitz, Feng Liu and Roger Patient Int. J. Dev. Biol. (2010) 54: 1139 - 1149

The mob as tumor suppressor (mats1) gene is required for growth control in developing zebrafish embryos

Yuan Yuan, Shuo Lin, Zuoyan Zhu, Wenxia Zhang and Zhi-Chun Lai

Int. J. Dev. Biol. (2009) 53: 525-533

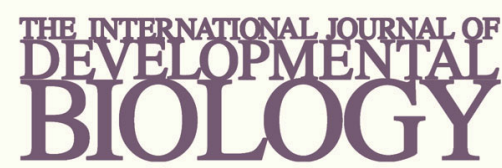

Volume 54 Nos. 6/7

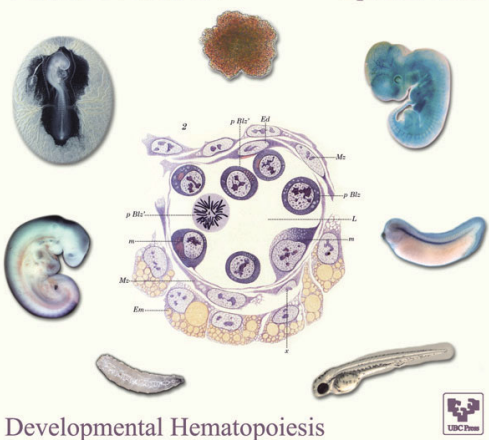

5 yr ISI Impact Factor $(2011)=2.959$
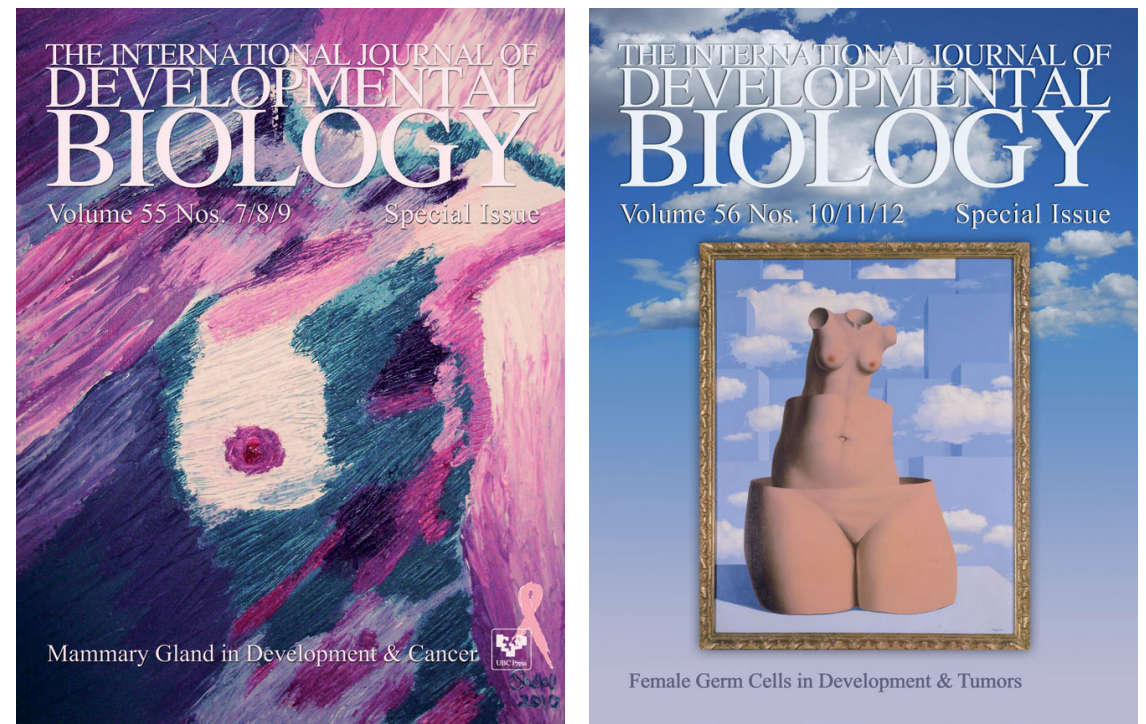

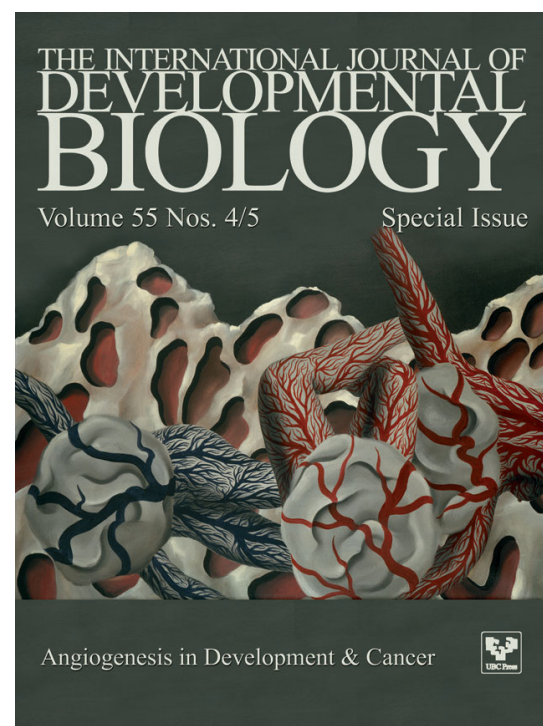

